\title{
Comparison of a traditional systematic review approach with review-of-reviews and semi-automation as strategies to update the evidence
}

\author{
Shivani M. Reddy ${ }^{1 *}$, Sheila Patel ${ }^{2}$, Meghan Weyrich³, Joshua Fenton ${ }^{3}$ and Meera Viswanathan ${ }^{2}$
}

\begin{abstract}
Background: The exponential growth of the biomedical literature necessitates investigating strategies to reduce systematic reviewer burden while maintaining the high standards of systematic review validity and comprehensiveness.

Methods: We compared the traditional systematic review screening process with (1) a review-of-reviews (ROR) screening approach and (2) a semi-automation screening approach using two publicly available tools (RobotAnalyst and AbstrackR) and different types of training sets (randomly selected citations subjected to dual-review at the title-abstract stage, highly curated citations dually reviewed at the full-text stage, and a combination of the two). We evaluated performance measures of sensitivity, specificity, missed citations, and workload burden

Results: The ROR approach for treatments of early-stage prostate cancer had a poor sensitivity (0.54) and studies missed by the ROR approach tended to be of head-to-head comparisons of active treatments, observational studies, and outcomes of physical harms and quality of life. Title and abstract screening incorporating semi-automation only resulted in a sensitivity of $100 \%$ at high levels of reviewer burden (review of 99\% of citations). A highly curated, smaller-sized, training set $(n=125)$ performed similarly to a larger training set of random citations $(n=938)$.

Conclusion: Two approaches to rapidly update SRs—-review-of-reviews and semi-automation—failed to demonstrate reduced workload burden while maintaining an acceptable level of sensitivity. We suggest careful evaluation of the ROR approach through comparison of inclusion criteria and targeted searches to fill evidence gaps as well as further research of semi-automation use, including more study of highly curated training sets.
\end{abstract}

\section{Background}

Many clinical guideline and policy statements rely on a systematic evidence review (SR) that synthesizes the evidence base. SRs are labor-intensive projects due to high standards of validity and comprehensiveness. Guidance from the Institute of Medicine and the Effective Health Care (EHC) Program favor high sensitivity of literature searches and screening over specificity. All citations

\footnotetext{
*Correspondence: sreddy@rti.org

${ }^{1}$ RTI International, 307 Waverly Oaks Road, Suite 101, Waltham, MA 02452, USA

Full list of author information is available at the end of the article
}

require dual review of abstracts and articles at each step of screening to identify and include all relevant research in the SR [1-3]. With the exponential growth of biomedical literature, a sensitive search can yield thousands of citations for dual abstract review and hundreds of articles for dual full-text review, whereas the number of articles ultimately included in the evidence review is typically much less.

In this study, we examine strategies to reduce workload burden of title and abstract screening as an adjunct investigation in parallel to conducting a traditional update SR on treatments for early-stage prostate cancer.

(C) The Author(s). 2020 Open Access This article is licensed under a Creative Commons Attribution 4.0 International License, which permits use, sharing, adaptation, distribution and reproduction in any medium or format, as long as you give appropriate credit to the original author(s) and the source, provide a link to the Creative Commons licence, and indicate if changes were made. The images or other third party material in this article are included in the article's Creative Commons licence, unless indicated otherwise in a credit line to the material. If material is not included in the article's Creative Commons licence and your intended use is not permitted by statutory regulation or exceeds the permitted use, you will need to obtain permission directly from the copyright holder. To view a copy of this licence, visit http://creativecommons.org/licenses/by/4.0/. The Creative Commons Public Domain Dedication waiver (http://creativecommons.org/publicdomain/zero/1.0/) applies to the data made available in this article, unless otherwise stated in a credit line to the data. 
Early-stage prostate cancer is a common but indolent disease that may remain clinically silent for a man's lifetime; thus, the decision to treat clinically localized prostate cancers balances the risk of common treatment side effects (such as urinary incontinence or erectile dysfunction) with the less common risk of cancer progression and death [4-6]. In the current example, we performed an update search on comparative effectiveness of treatments for early-stage prostate cancer, including patientcentered outcomes. We relied on two recent SRs of treatments for early-stage prostate cancers: one focused on comparisons of active treatment to conservative management [7], and the second also included head-to-head comparisons of active treatments [8]. These reviews were conducted in 2014 and 2016, necessitating a SR update. We identified three approaches to updating SRs: the traditional search and screening method recommended by the EHC Program, a "review of reviews" (ROR) approach, and, semi-automation of abstract screening. A traditional search approach involves conducting searches in multiple databases, including searches of databases, grey literature sources, as well as a ROR: an approach intensive in labor and time. The ROR approach involves identifying and selecting SRs to either use in their entirety or to rely on for identifying relevant primary studies. Because the yield of eligible systematic reviews is smaller than comprehensive database searches, the effort requires less labor and time than a traditional approach but has the potential to miss relevant citations. Semi-automation screening software uses text-mining algorithms to find patterns in unstructured text and machine learning (ML) to train predictive classification algorithms to make inclusion and exclusion decisions or prioritize relevant citations in the title and abstract screening step of an SR [9]. Semi-automation tools have the potential to alleviate the burden on human reviewers by decreasing the number of citations to be screened by human reviewers, replacing one of two human reviewers, or using screening prioritization to improve screening workflow [10]. Active learning is a type of ML where the algorithm and reviewer interact: the algorithm generates a list of prioritized citations for the researcher to review rather than presenting unscreened citations in a random order; the next step of reviewer inclusion and exclusion decisions further train the predictive model [11].

In this study, we compare the traditional SR screening process with (1) a ROR screening approach and (2) a semi-automation screening approach using two publicly available tools (RobotAnalyst and AbstrackR). With respect to semi-automation approaches, we evaluate training sets composed of randomly selected citations from the traditional database search, highly curated citations identified from full-text review, and a combination of the two to examine ways reviewers can practically incorporate ML tools into the review workflow. Similarly, ML algorithms predict a probability that a citation will be included in the final SR, and we examine different thresholds for inclusion to understand the trade-off between inclusion probability thresholds and sensitivity when incorporating ML into SRs. We evaluate performance measures of sensitivity, specificity, missed citations, and workload burden to assess if either approach could maintain the sensitivity of a traditional review approach while reducing the number of full-text articles a reviewer would have to read.

\section{Methods}

\section{Traditional review}

We first conducted the traditional database search from 1 January 2014 to 27 November 2018, beginning a few months prior to the most recent reviews on the topic [7, $8]$. We created search strings to replicate the yield and be consistent with the search criteria of the two more recent reviews related to the research. Appendix 1 lists our search strings for PubMed, Cochrane, and grey literature sources, specifically, HSRPproj, clinicaltrials.gov, and AHRQ and PCORI projects. Appendix 2 lists our inclusion and exclusion criteria. For studies identified through the database search, we dually and independently reviewed titles and abstracts against the inclusion and exclusion criteria. Articles flagged for inclusion by either reviewer then moved to full-text review. Each fulltext article was then dually and independently reviewed. Consensus or third-party adjudication resolved conflicts. The results of the ROR (described below) were included in the final yield of the traditional review.

\section{Review of reviews approach}

For the ROR, two team members independently selected SR citations from the database search for identification of primary research studies for further title and abstract screening and potential full-text review. The same team members conducted both the traditional search and the hand search to ensure greater consistency for the traditional search and ROR. The results of the ROR approach (i.e., ROR alone) were compared to the traditional review approach (i.e., database search and ROR).

\section{Semi-automation approach}

For the semi-automation approach, we used two software programs that apply machine learning for title and abstract screening and are readily available for current use. RobotAnalyst is publicly available, web-based software, developed by the National Centre for Text Mining, with a growing evidence base across several topic areas $[12,13]$. After reviewers screen at least 25 citations, the reviewer can train a ML algorithm to predict the 
probability that an unlabeled citation should be included. Each unlabeled citation is assigned an inclusion prediction probability (0 to 1.0). The review team can assign a threshold for the inclusion probability, above which citations are included and below which they are excluded, to reduce the number of citations that need to be manually reviewed. AbstrackR is an open-source tool for facilitating the citation screening process that has an active learning setting designated at the start of the review. After researchers screen a certain number of randomly selected citations, the active learning algorithm assigns a score to unlabeled citations analogous to the inclusion prediction generated by RobotAnalyst, and selects the next citation for the reviewer to screen based on a priority ranking table stored in the project database. AbstrackR periodically calls on the ML library to refine the ML algorithm and re-rank the unlabeled citations based on additional human screening decisions. The AbstrackR active learning system performs citation re- prioritization asynchronously from human review due to the computational cost, which could slow down the program and impede efficient expert review [14].

These two tools were chosen for their public availability. AbstrackR, specifically, is supported by the Agency for Healthcare Research and Practice, free to users, and is a familiar interface to Evidence-based Practice Centers for title-abstract screening. Our tests of each program included (1) creating training sets of dually reviewed abstracts, (2) invoking ML to predict inclusion probabilities of the unlabeled citations, and (3) comparing the semiautomated approach to a traditional review approach.

\section{RobotAnalyst}

For the RobotAnalyst tests (Fig. 1), we created three training sets. We present these in order of the size and accuracy of the training set. The first training set included up to $30 \%$ of all citations retrieved from PubMed and Cochrane Library database searches. An excel

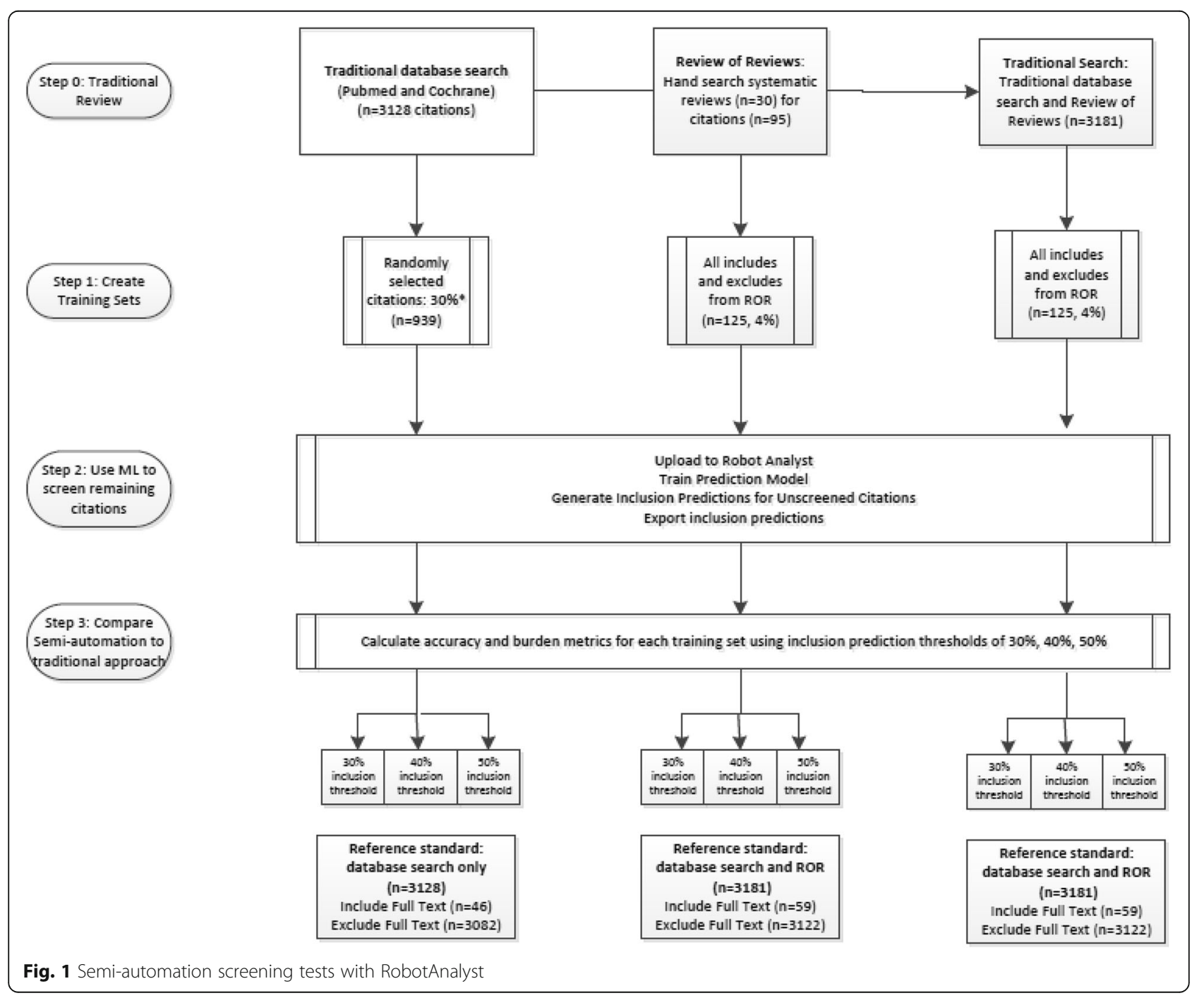


random number generator was used to select $30 \%$ of dually screened citations from the traditional review $(n=$ 939). Prior studies using smaller sized training sets (< $20 \%)$ have shown poor sensitivity $[15,16]$. At least $5 \%$ of the citations that were included after title and abstract screening in the traditional review were included in the training set to ensure the algorithm was exposed to citations that would be included after the first stage of screening. This training set simulates prospective incorporation of ML into title-abstract screening. The training set labels are based on decisions made after dual review of title and abstracts only; thus, studies included after this first screening step may be true positives or false positives, the latter excluded after full-text review.

The second training set used inclusion decisions from the ROR ( $n=125,4 \%)$, specifically inclusion after fulltext review. The advantage of this training set is that it contains citations that have been dually reviewed in two stages and does not include any false positives (i.e., studies screening positive at title-abstract review but negative at full-text review). This training set involves greater effort in generating the curated ROR set and simulates combining an established rapid review method with semi-automation.

The third training set combined the ROR training set and $30 \%$ randomly chosen citations with full-text decisions from the traditional review (versus title-abstract decisions). This last training set builds on the ROR training set and, as with the ROR training set, there are no false-positive labels used to train the ML algorithm. This strategy is closest in workflow to traditional systematic review processes that do not employ semiautomation, while potentially reducing the number of citations to screen by over half, though does require the greatest effort to create the training set.

The training sets were uploaded to RobotAnalyst, the reviewer explicitly called on RobotAnalyst to train the prediction model and update unlabeled citations with an inclusion prediction probability, and the probabilities were exported from RobotAnalyst for analysis. To compare a semi-automated approach to the traditional review approach, we chose three thresholds for the inclusion prediction probability: $30 \%, 40 \%$, and $50 \%$. The default setting in RobotAnalyst is $50 \%$, and we examine two lower thresholds to assess the impact on sensitivity and reviewer burden.

\section{AbstrackR}

For the AbstrackR semi-automation test, a simulation program was used to estimate outcomes for multiple training sets, beginning with 500 randomly selected citations and ranging up to 3100 citations. (Figure 2) Because active learning used by Abstrack $\mathrm{R}$ is a dynamic process that occurs asynchronously from the time of screening, the initial training set of random citations and order of subsequent citations presented to the reviewer can influence final results. Thus, for each training set, 50 iterations of the simulation were performed for each training set, selecting a different set of random citations and allowing the algorithm to pick the next 50 citations that were predicted to be most likely relevant for human review. Citations with predictions falling below 0.40 were considered excludes by AbstrackR.

\section{Outcomes}

Figure 3 summarizes the outcomes evaluated: sensitivity, specificity, missed citations, burden, and time saved. Time savings was calculated using an estimate of $30 \mathrm{~s}$ per abstract review by an experienced reviewer, as previously cited in the literature; our experience is also consistent with this estimate [16]. For the ROR approach, we further compared the inclusion criteria for the update SR with the inclusion criteria of the SRs included in the ROR to assess how well our PICOTS are represented by the ROR approach. We then describe citations missed by the ROR approach to better understand if certain study characteristics are prone to being missed by rapid review approaches.

We present results for the ROR and semi-automation approaches compared with the traditional approach. Outcomes for the semi-automation approach can be calculated for the entire process-dual human review of the training set and incorporating ML to predict inclusion of remaining citations-or for the performance of the ML algorithm alone. We present the outcomes for the entire process as our main findings because it best represents how semi-automation would be employed in current practice. (Outcomes for the ML algorithm alone are presented in Appendix 3.) Figure 3 shows the confusion matrix used to calculate outcomes incorporating the training set and ML inclusion decisions. We assumed that there were no false-negative inclusion decisions made by human reviewers. Outcomes reported for the AbstrackR semi-automation test include sensitivity and burden, also based on the entire review process as discussed above, though each outcome measure is an average of the 50 iterations performed for each of the 27 training sets and we were unable to estimate missed citations. Because AbstrackR uses active learning, we are unable to estimate outcomes for the algorithm alone, as remaining citations are not a random subset of unlabeled citations.

\section{Results}

Review of reviews approach

Figures 4 and 5 show the results of our traditional search and ROR. The traditional database search yielded 3128 citations, with 46 studies included after full-text 


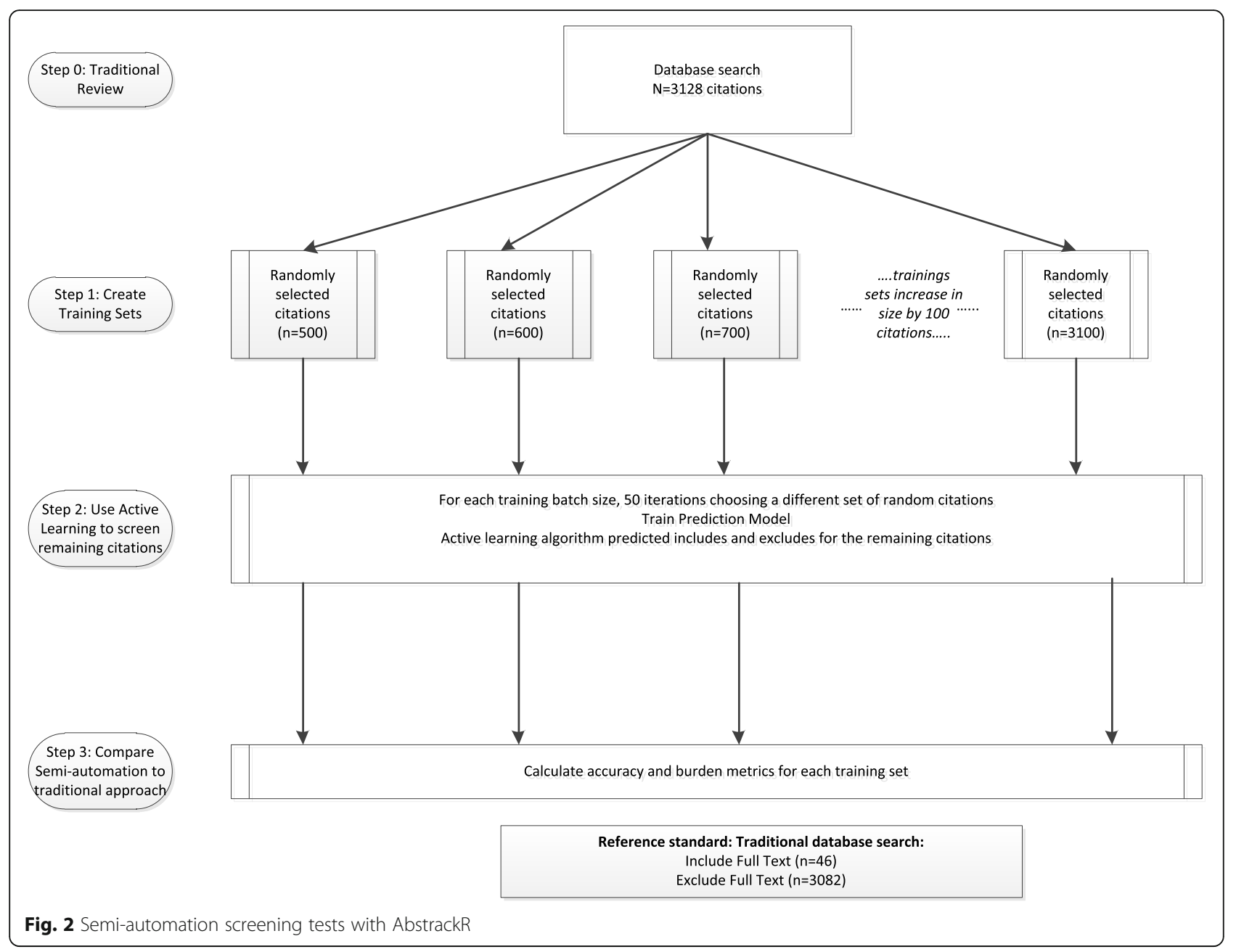

screening. The ROR identified 30 systematic evidence reviews, and 95 primary studies were retrieved after a hand search. Among the 125 studies identified for screening in the ROR, 33 were included after full-text screening. The $2 \times 2$ matrix comparing the ROR approach to the traditional search is shown in Table 1. The ROR approach greatly reduced the number of citations screened ( $n=125$ vs. 3181 , burden $4 \%$, time savings $25.5 \mathrm{~h}$ ), though had a low sensitivity (56\%) and 26 missed citations. Table 2 summarizes the update SR inclusion criteria and overlapping inclusion criteria of SRs found in the ROR approach. Among SRs included in the ROR approach, inclusion criteria for population were well-matched to the update SR, apart from three studies that included $>10 \%$ of men with locally invasive or advanced disease. Most SRs included surgery or radiation treatment arms, active comparators, and randomized or non-randomized trials in their inclusion criteria. Ablative therapies as well as observational studies (cohorts and case control studies) and single arm study designs were less likely to be listed as SR inclusion criteria.
Table 3 presents characteristics of studies missed by the ROR approach search. We observe that a significant number of missed citations are of active therapies or comparisons of active therapies. Over $50 \%$ of missed citations were published in 2016 or later $(n=16)$; among this subset of more recent studies, 11 studies had an active treatment comparator. Missed studies were much more likely to be observational studies versus trials $(88$ vs. $8 \%$ ). Among the 25 uniquely missed studies, 16 were cohort studies (Appendix 4). The outcome of interest in $75 \%$ of the missed cohort studies were harms-qualityof-life outcomes measuring urinary or erectile dysfunction, psychological harms such as mental health disease or decision regret, and procedure-or medicationrelated harms.

\section{Semi-automation approach: RobotAnalyst}

The results of the semi-automation test using RobotAnalyst with a training set of random citations (Table 4) illustrated higher sensitivity for lower inclusion probability thresholds $(100 \%$ for $30 \%$ inclusion probability 


SENSITIVITY: The proportion of records correctly identified as relevant by ROR or semi-automation out of
the total deemed relevant by the human reviewers (following full text screening)
Sensitivity = (true positives) / (true positives + false negatives)
SPECIFICITY: The proportion of records correctly identified as irrelevant by ROR or semi-automation out of
the total deemed irrelevant by the human reviewers (following full text screening)
Specificity = (true negatives) / (true negatives + false positives)

Fig. 3 Outcome definitions and confusion matrix

threshold vs. $74 \%$ for $50 \%$ inclusion probability threshold), with no missed citations at a threshold of $30 \%$ and 12 missed citations at a threshold of $50 \%$. However, at lower inclusion probability thresholds, specificity decreased (30\% vs 55\%) and the time saved was minimal (11 min for threshold 30\%), as the algorithm at this threshold predicted that nearly all of the citations would be included $(2168 / 2190=99 \%)$.

The semi-automation test using RobotAnalyst with the ROR training set showed similar trends of increased sensitivity at lower thresholds of inclusion probability $(100 \%$ sensitivity for $30 \%$ inclusion probability threshold vs. $69 \%$ sensitivity for $50 \%$ inclusion probability threshold), though higher reviewer burden and minimal time saved (99\% and $8 \mathrm{~min}$, respectively) (Table 5). Compared to the ROR approach alone, the semi-automation approach using the ROR citations as the training set improved sensitivity and missed fewer citations even at the higher inclusion probability threshold. For example, for a threshold of 0.5 , semi-automation using the ROR citations as a training set missed 18 citations compared with 26 citations.

Compared to the training set of random citations $(n=$ 938), the ROR training set was considerably smaller $(n=$ 125), though demonstrated similar performance in terms of sensitivity and missed citations, as well as similar estimates of burden and time savings. Plotting sensitivity against burden shows that the semi-automation approach using a ROR training set performs slightly better than using a random dataset (Fig. 6). The third training set combined the ROR training set with a random set of citations, labeled with full-text decisions. The results of the semi-automation test for this training set were similar to the ROR test alone (Table 6, Fig. 6).

\section{Semi-automation approach: AbstrackR}

The semi-automation test of AbstrackR illustrated similar findings of increased sensitivity with larger training sets but increasing burden as well (Fig. 7, Appendix 5). For a training set of approximately $30 \%$ of the total 


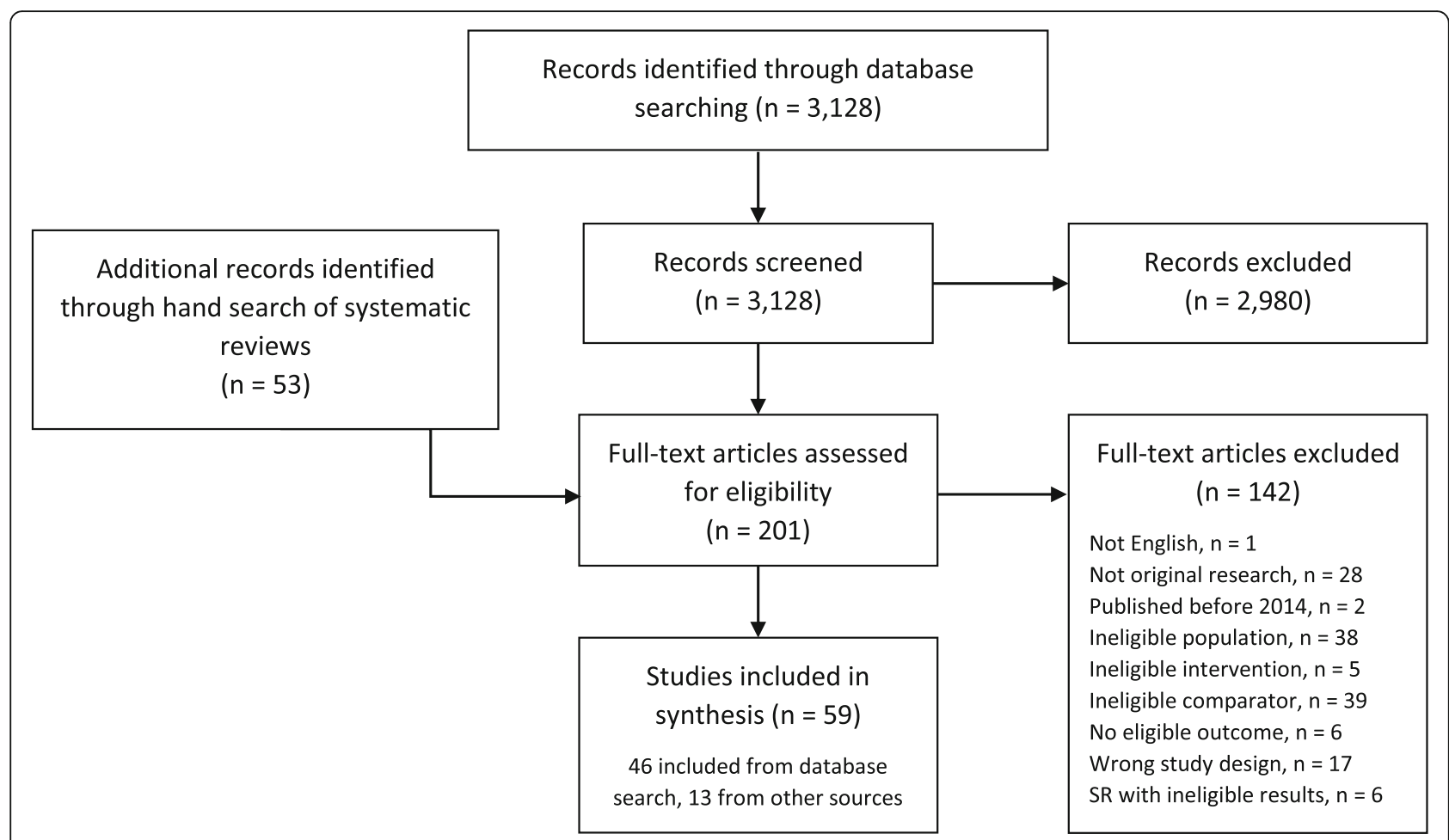

Fig. 4 Traditional approach PRISMA

citations, the mean sensitivity was $77 \%$ and the mean burden was $76 \%$. Sensitivity did not exceed $90 \%$ until the training set includes 2500 of the 3128 citations.

\section{Discussion}

In this study, we evaluated two strategies-a review of review approach and semi-automation-to update the most recent SRs on treatments of early prostate cancer. Review of reviews are a commonly employed strategy for rapid reviews [17] The ROR approach for treatments of early-stage prostate cancer had a poor sensitivity (0.56) and studies missed by the ROR approach tended to be of head-to-head comparisons of active treatments, observational studies, and more recent studies (as would be expected given the lag between literature searches and publication). Even though active comparators were part of the inclusion criteria in the majority of SRs in the ROR, 15 of the 25 missed citations (60\%) included an active treatment comparison arm. Among missed studies with an active comparator, 11 studies were published in 2016 or later, suggesting comparative effectiveness studies may be missed due to the delay between SR search and publication. Approximately two-thirds of SRs captured by the ROR approach included trials as an eligible study design; only one missed citation was a trial. However, less than half of SRs included observational studies, and this study design accounted for nearly $90 \%$ of missed citations. Many evidence grading systems like
GRADE have downgraded quality of observational studies [18] in the past, which can impact overall strength of evidence rating of an SR. Reviewers may decide to limit SR inclusion criteria to the highest quality research (i.e., trials) and exclude observational studies, which can also limit the search yield to a more manageable number of citations for a SR team. As a consequence of excluding observational study designs, quality of life and physical harms were among the most common missed outcomes in the ROR approach, which has implications for projects that focus on important patient-centered outcomes. Our results suggest careful consideration before employing a ROR approach to expedite the review process. Planning a priori to compare the inclusion criteria of a proposed ROR with those of retrieved SRs may help to proactively identify study characteristics for targeted primary literature searches.

The promise of ML to reduce reviewer burden of sometimes tedious SR steps like title and abstract screening has yet to be realized [10, 19]. Many prior studies have retrospectively evaluated the performance of ML tools, with knowledge of the final inclusion and exclusion decisions, which reviewers cannot know prospectively. In this study, we simulated three possible prospective uses of semi-automation: incorporating ML during title-abstract screening, adding ML to a ROR, and applying ML after conducting a ROR and a partial title-abstract and full-text review. By using a training set 


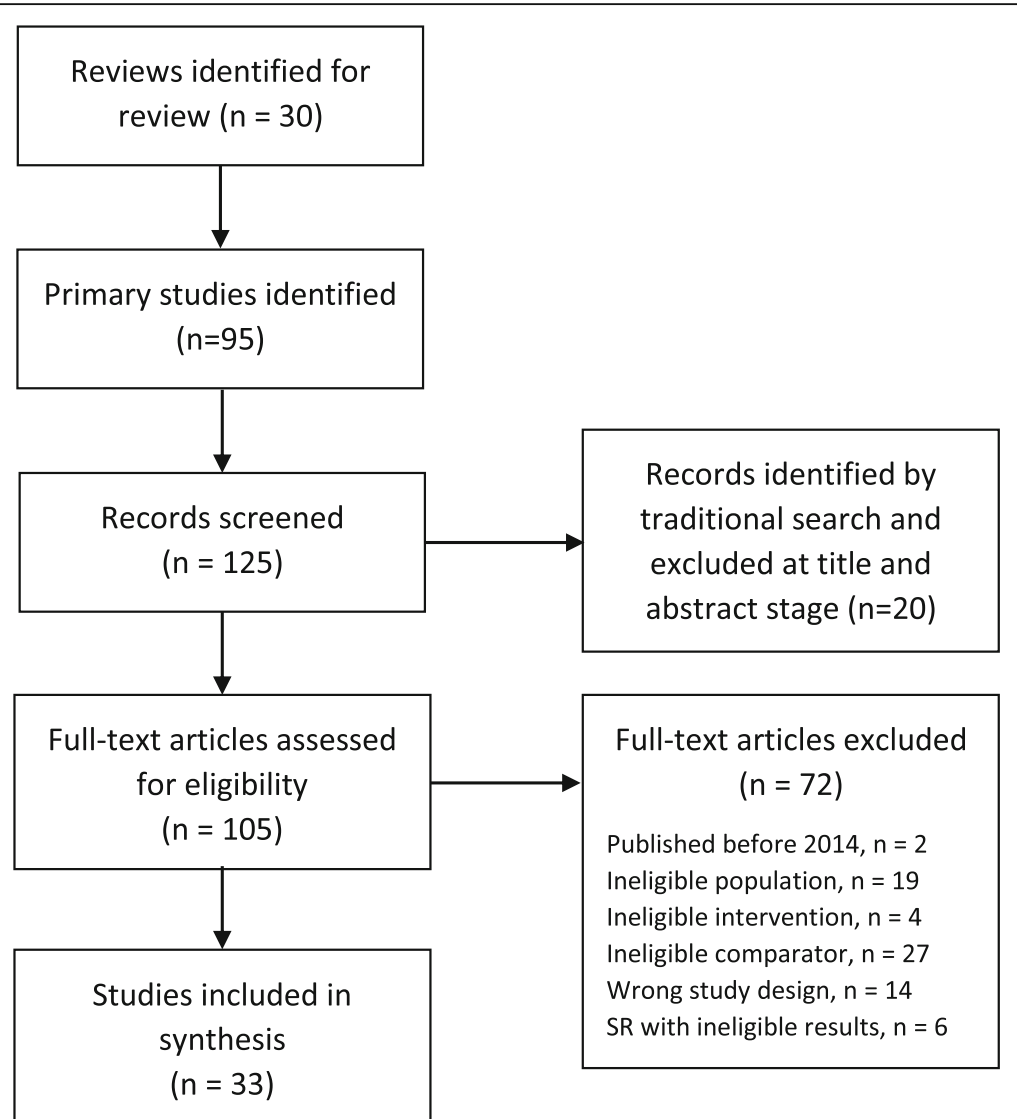

Fig. 5 Review of reviews PRISMA

of random citations with labels determined by titleabstract screening, we simulated incorporation of ML at the title-abstract stage, where many citations labeled as included will go on to be excluded after full-text screening. In this SR update of early prostate cancer treatments, 148 abstracts from the database search were included after title and abstract screening and only 46 (31\%) were included in the final SR update. Both tests of RobotAnalyst and AbstrackR using random citations with labels from title and abstract screening demonstrated acceptable sensitivity only at the high levels of

Table 1 Sensitivity and specificity of review of reviews approach compared with traditional review approach

\begin{tabular}{|c|c|c|c|c|}
\hline & & \multicolumn{2}{|c|}{ Traditional review approach } & \multirow[b]{2}{*}{ Total } \\
\hline & & Include & Exclude & \\
\hline \multirow{4}{*}{$\begin{array}{l}\text { Review of } \\
\text { reviews approach }\end{array}$} & Include & 33 & 0 & 33 \\
\hline & Exclude & 26 & 3122 & 3148 \\
\hline & Total & 59 & 3122 & 3181 \\
\hline & & $\begin{array}{l}\text { Sensitivity } \\
0.56\end{array}$ & $\begin{array}{l}\text { Specificity } \\
1.00\end{array}$ & \\
\hline
\end{tabular}

*Of the 3122 excluded studies, 92 were excluded after full-text review and 3030 were citations not retrieved through the review of reviews approach burden that would not effectively reduce the workload compared to a traditional review. Our findings for AbstrackR tests mirrored RobotAnalyst tests in that increasing sensitivity was associated with increased reviewer burden. Unlike previous AbstrackR simulations, where the mean sensitivity curve rises quickly and flattens out at a high sensitivity at lower levels of burden, the sensitivity and burden curves had similar slopes [11]. This may have been due to this review having a small proportion of included studies (4\%).

For semi-automation tests using the ROR training set and ROR with partial full-text review, we invested additional effort to create training sets for RobotAnalyst that only included labels determined after dual full-text review. We observed improved sensitivity of the MLenhanced process for the same workload using a much smaller training set of curated citations $(n=125)$ compared with a training set of random citations $(n=938)$. (Figure 6) Removing false-positive labels applied at the title-abstract screening stage may reduce "noise" in the training set and more effectively train the ML algorithm [15]. Adding roughly a third of the search results with full-text inclusion labels to the ROR training set did not improve sensitivity much over the smaller training set. 
Table 2 Comparison of inclusion criteria for the systematic review update and systematic reviews in review of reviews (ROR) approach

\begin{tabular}{|c|c|c|c|}
\hline & \multirow[t]{2}{*}{$\begin{array}{l}\text { Systematic review update Inclusion } \\
\text { criteria }\end{array}$} & \multicolumn{2}{|c|}{$\begin{array}{l}\text { SRs with inclusion criteria matching SR update eligibility } \\
\text { criteria* }\end{array}$} \\
\hline & & $N$ & $\%$ \\
\hline Population & Localized prostate cancer & 27 & 90 \\
\hline \multirow[t]{2}{*}{ Interventions studied in one or more arm } & Active therapy (surgery and/or radiation) & 17 & 57 \\
\hline & Ablative therapy only & 9 & 30 \\
\hline \multirow[t]{3}{*}{ Comparators studied in one or more arm } & Active treatment comparator & 17 & 57 \\
\hline & Conservative treatment comparator & 11 & 37 \\
\hline & No comparator & 8 & 27 \\
\hline \multirow[t]{3}{*}{ Study design } & Randomized and non-randomized trials & 20 & 67 \\
\hline & Observational studies & 14 & 47 \\
\hline & Single arm studies & 8 & 27 \\
\hline Total & & 30 & 100 \\
\hline
\end{tabular}

*Studies may include multiple arms or multiple study types; percentages do not sum to 100

Prior studies have suggested that ML algorithms may perform better with training sets that are more balanced and less well in collections with a smaller proportion of includes $[15,16]$.

Our results suggest that the increased effort to create a more curated training set may reduce downstream burden; however, there remain several questions on how to include ML in SR processes. ML algorithms predict the probability of inclusion for a given citation, but it is unclear if and when human reviewers can safely stop screening without missing relevant studies. Even if a citation has a low inclusion probability, it may still be relevant [13, 20]. Even if a reviewer screens all citations using ML predictions to prioritize more relevant citations first, there is a risk of user complacency, agreeing with the prediction rather than screening randomly presented abstracts independently free of bias [13]. The content of the SR may also affect performance of a

Table 3 Characteristics of studies missed by the review of reviews approach

\begin{tabular}{|c|c|c|c|}
\hline \multirow[t]{2}{*}{ Sample size } & \multirow[t]{2}{*}{ Subtype } & \multicolumn{2}{|c|}{ Studies missed by the ROR search* } \\
\hline & & $N$ & $\%$ \\
\hline \multirow[t]{3}{*}{ Interventions studied in one or more arm } & Surgery & 15 & 60 \\
\hline & Radiation therapy & 15 & 60 \\
\hline & Ablative therapy & 10 & 40 \\
\hline \multirow[t]{3}{*}{ Comparators } & Active treatment comparator & 15 & 60 \\
\hline & Conservative treatment comparator & 8 & 32 \\
\hline & No comparator & 8 & 32 \\
\hline \multirow[t]{3}{*}{ Study design } & Randomized and non-randomized trials & 1 & 4 \\
\hline & Observational studies & 24 & 88 \\
\hline & Single arm studies or within-treatment comparisons ${ }^{\dagger}$ & 2 & 8 \\
\hline \multirow[t]{5}{*}{ Year of publication } & 2014 & 3 & 12 \\
\hline & 2015 & 6 & 24 \\
\hline & 2016 & 7 & 28 \\
\hline & 2017 & 8 & 32 \\
\hline & 2018 & 1 & 4 \\
\hline Total & & $25^{\prime \prime}$ & 100.0 \\
\hline
\end{tabular}

*Studies may have multiple arms; percentages do not sum to 100

${ }^{\dagger}$ Single-arm studies (or within-treatment comparisons) were eligible only for ablative therapies

"The ROR approach missed a total of 26 eligible citations, which one was a companion study and one was the Fenton et al. review. The analysis is limited to the 25 primary studies

Abbreviations: $N$ number, NA not applicable, $R C T$ randomized controlled trial, $R O R$ review of reviews 
Table 4 Semi-automation test with RobotAnalyst using a training set of dually reviewed randomly selected citations with labels from title and abstract screening

\begin{tabular}{|c|c|c|c|}
\hline & \multicolumn{3}{|c|}{$\begin{array}{l}\text { Traditional database search } \\
\text { Total citations: } 3128 \\
\text { Title-abstract screening: } 148 \text { includes/2980 excludes } \\
\text { Full-text screening: } 46 \text { includes/3082 excludes }\end{array}$} \\
\hline & \multicolumn{3}{|c|}{$\begin{array}{l}\text { Training set } \\
\text { Labeled citations: } 938(30 \%) \\
\text { Training set labels: TP (15), FP (32), TN (891) } \\
\text { Unlabeled citations assigned inclusion prediction by ML algorithm: } 2190\end{array}$} \\
\hline & Inclusion prediction: 0.3 & Inclusion prediction: 0.4 & Inclusion prediction: 0.5 \\
\hline Predicted includes & 2168 & 1970 & 1363 \\
\hline Predicted excludes & 22 & 220 & 827 \\
\hline Sensitivity & $100 \%$ & $93 \%$ & $74 \%$ \\
\hline Specificity & $30 \%$ & $36 \%$ & $55 \%$ \\
\hline Missed citations & 0 & 3 & 12 \\
\hline Burden & $99 \%$ & $93 \%$ & $74 \%$ \\
\hline Time savings (min) & 11 & 110 & 413.5 \\
\hline
\end{tabular}

FP false positive, $M L$ machine learning, $T P$ true positive, $T N$ true positive

semi-automation approach. Our review included a breadth of interventions and study designs, including observational studies. A prior study evaluating ML in multiple SRs found that machine learning supported screening had improved sensitivity for SRs including only randomized controlled trails versus studies including observational studies like case series [16]. Another study found poorer sensitivity of semi-automation approaches for SRs including multiple study populations, such as young adults and adults [15].

Strengths of this study include evaluation of the ROR approach, a commonly used strategy to expedite the SR process, and simulation of possible prospective uses of semi-automation, particularly using the results of the ROR as a training set for semi-automated screening. Prior studies have suggested using the original SR as the training set for an update [21], though an SR update may have a different set of PICOTS than the prior review due to shifts in the population of interest, greater availability of diagnostic tools like biomarkers, advances in treatment options, and greater interest in patientcentered outcomes. Citations from a ROR train the machine learning algorithm with currently relevant data.

Limitations of the study include using a training set of random citations that were not truly random as we included at least $5 \%$ of citations that would have been

Table 5 Semi-automation test with RobotAnalyst using a training set of dually reviewed citations from a review-of-review with labels from full-text screening

\begin{tabular}{|c|c|c|c|c|}
\hline & \multicolumn{4}{|l|}{$\begin{array}{l}\text { Traditional search } \\
\text { Total citations: } 3181 \\
\text { Title-abstract screening: } 201 \text { includes/2980 excludes } \\
\text { Full-text screening: } 59 \text { includes/3122 excludes } \\
\end{array}$} \\
\hline & \multirow{2}{*}{$\begin{array}{l}\text { Review of reviews } \\
\text { Total SR citations: } 30 \\
\text { Total primary study citations: } 95 \\
\text { Title-abstract screening: } 76 \text { includes/49 excludes } \\
\text { Full-text screening } 33 \text { includes/43 excludes } \\
\text { Number of citations not screened: } 3030\end{array}$} & \multicolumn{3}{|c|}{$\begin{array}{l}\text { Training set: ROR citations } \\
\text { Labeled citations: } 125(4 \%) \\
\text { Training set labels: TP (33), FP (0), TN (92) } \\
\text { Unlabeled citations assigned inclusion prediction by ML algorithm: } 3056\end{array}$} \\
\hline & & Inclusion prediction: 0.3 & Inclusion prediction: 0.4 & Inclusion Prediction: 0.5 \\
\hline Predicted includes & NA & 3040 & 2819 & 1166 \\
\hline Predicted excludes & NA & 16 & 237 & 1890 \\
\hline Sensitivity & $54 \%$ & $100 \%$ & $97 \%$ & $69 \%$ \\
\hline Specificity & $100 \%$ & $3 \%$ & $10 \%$ & $63 \%$ \\
\hline Missed citations & 26 & 0 & 2 & 18 \\
\hline Burden & $4 \%$ & $99 \%$ & $93 \%$ & $41 \%$ \\
\hline Time savings (min) & 1561 & 8 & 118.5 & 945 \\
\hline
\end{tabular}

FP false positive, $M L$ machine learning, NA not applicable, $T P$ true positive, $T N$ true positive 
Table 6 Semi-automation test with RobotAnalyst using a training set of dually reviewed citations from a review-of-review and randomly selected citations with labels from full-text screening

\begin{tabular}{|c|c|c|c|}
\hline & \multicolumn{3}{|c|}{$\begin{array}{l}\text { Traditional search } \\
\text { Total citations: } 3181 \\
\text { Title-abstract screening: } 201 \text { includes/2980 excludes } \\
\text { Full-text screening: } 59 \text { includes/3122 excludes }\end{array}$} \\
\hline & \multicolumn{3}{|c|}{$\begin{array}{l}\text { Training set: ROR citations + } \mathbf{3 0} \% \text { random citations } \\
\text { Labeled citations: } 1063(33 \%) \\
\text { Training set labels: TP (40), FP (0), TN (1023) } \\
\text { Unlabeled citations assigned inclusion prediction by ML algorithm: } 2118\end{array}$} \\
\hline & Inclusion prediction: 0.3 & Inclusion prediction: 0.4 & Inclusion prediction: 0.5 \\
\hline Predicted includes & 2094 & 1765 & 676 \\
\hline Predicted excludes & 24 & 353 & 1442 \\
\hline Sensitivity & $98 \%$ & $84 \%$ & $80 \%$ \\
\hline Specificity & $34 \%$ & $44 \%$ & $79 \%$ \\
\hline Missed citations & 1 & 3 & 12 \\
\hline Burden & $99 \%$ & $89 \%$ & $55 \%$ \\
\hline Time savings (min) & 12 & 177 & 721 \\
\hline
\end{tabular}

$F P$ false positive, $M L$ machine learning, $T P$ true positive, $T N$ true positive

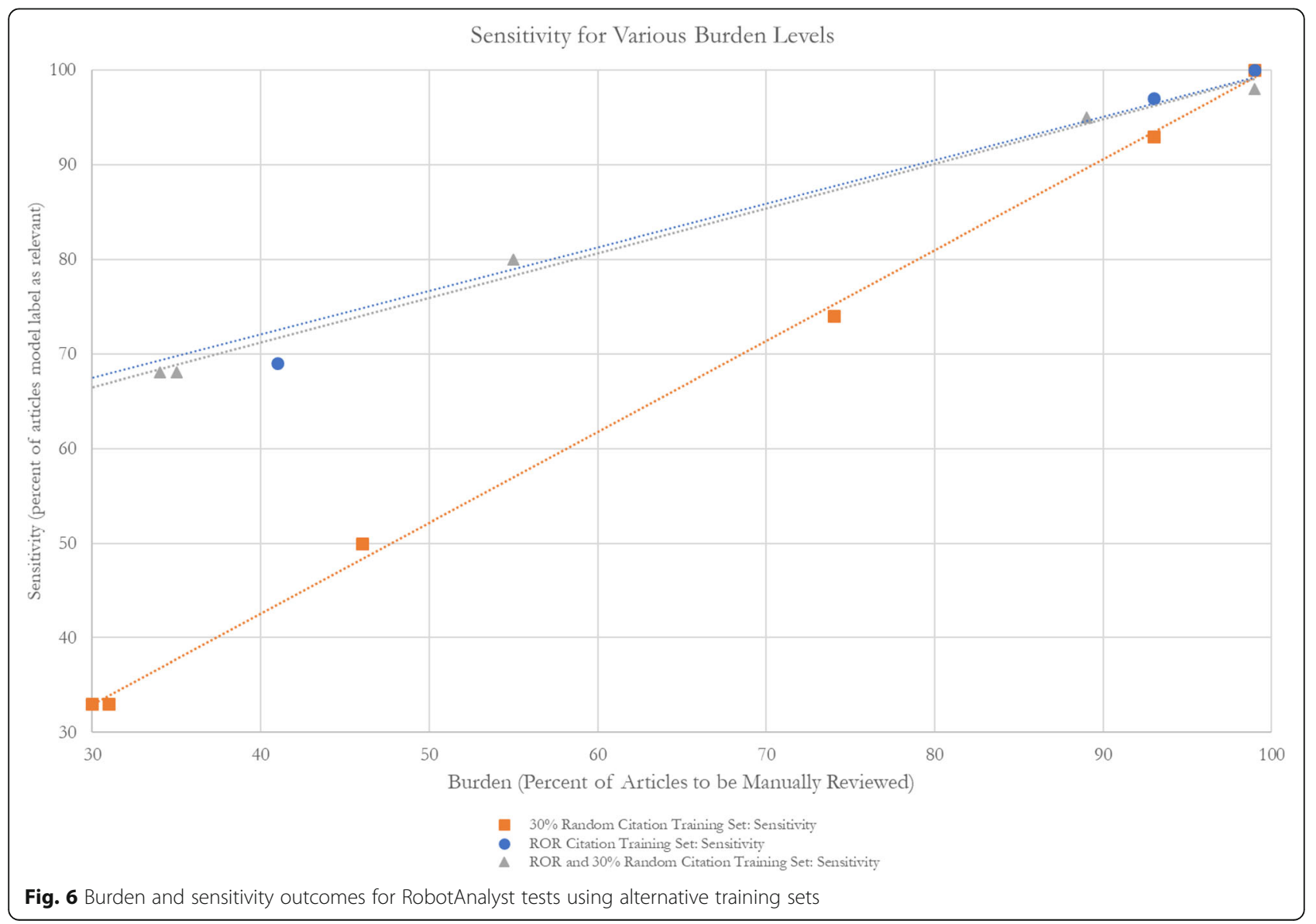




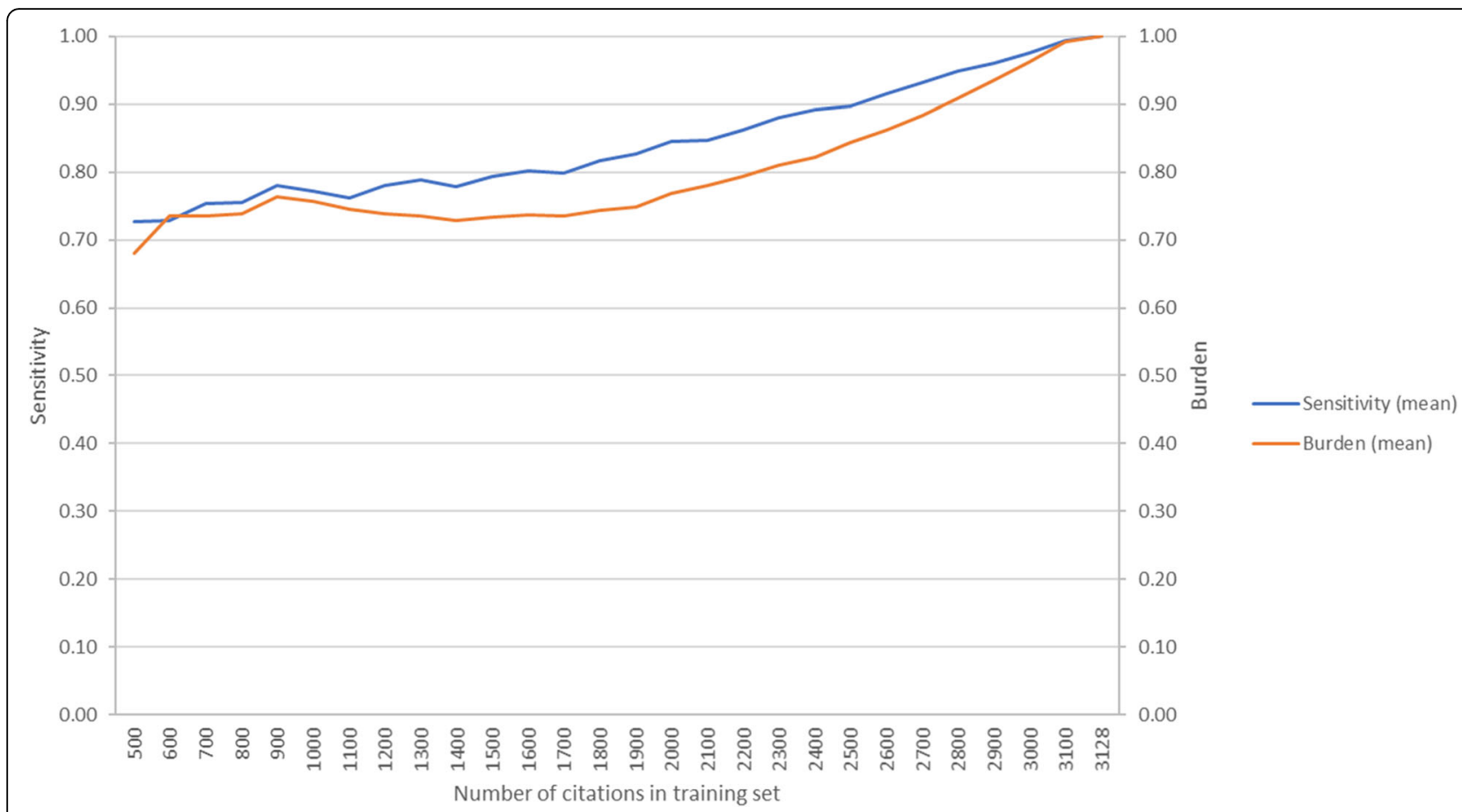

Fig. 7 Sensitivity and burden using AbstrackR active learning for title-abstract screening

included after title-abstract screening to ensure that the ML algorithm would be exposed to citations in the minority class. With the AbstrackR test, we had the capacity to perform 50 random training sets for multiple sizes and found comparable sensitivity and burden for a similarly sized training set used for the RobotAnalyst test. The time savings estimate may be lower than we found as abstracts screened earlier in title and abstract screening may require longer review than those screened towards the end. For both approaches-ROR and semiautomation, we must consider the generalizability of our results to other content areas and research questions. There is a risk of incorporation bias because the ROR is a subset of the gold standard of a traditional review, though the direction of this bias is to inflate sensitivity and specificity. Even with possible incorporation bias, the sensitivity and specificity of the ROR was poor. Finally, we used the traditional SR approach of dual abstract screening as the reference against which more expedient approaches of ROR and ML were compared. However, independent, dual review of abstracts does not preclude screening errors [22].

In conclusion, two approaches to rapidly update SRs-review-of-reviews and semi-automation-failed to demonstrate reduced workload burden while maintaining an acceptable level of sensitivity. We suggest careful evaluation of the ROR approach through comparison of inclusion criteria and targeted searches to fill evidence gaps as well as further research of semi- automation use, including more study of highly curated training sets, stopping rules for human screening, ML at the search stage to reduce yields, and prospective use of ML to reduce human burden in systematic evidence review.

\section{Supplementary information}

Supplementary information accompanies this paper at https://doi.org/10. 1186/s13643-020-01450-2.

Additional file 1: Appendix Table 1. PubMed search string and yield (11/27/2019). Appendix Table 2. Cochrane search string and yield (11/ 27/2019). Appendix Table 3. AHRQ Evidence Reports and Technology Assessments Search String and Yield (1/7/2019). Table 4. Drug

Effectiveness Review Project Drug Class Reviews Search String and Yield (1/7/2019). Table 5. National Guidelines Search String and Yield (1/7/ 2019). Table 6. ClinicalTrials.gov Search String and Yield (1/10/2019). Table 7. HSRPproj Search String and Yield (1/10/2019). Table 8. PCORI Portfolio Search String and Yield (1/10/2019). Appendix 2: Inclusion and Exclusion Criteria. Appendix 3. Outcomes for Machine Learning Algorithm Only. Table 3A. Semi-automation test with RobotAnalyst using a Training Set of Randomly-Selected Dually-Reviewed Citations with Labels from Title and Abstract Screening: Outcome Metrics for Machine Learning Algorithm Only. Table 3B. Semi-automation test with RobotAnalyst using a Training Set of Dually-Reviewed Citations from a Review-of-Review with Labels from Full Text Screening: Outcome Metrics for Machine Learning Algorithm Only. Table 3C. Semi-automation test with RobotAnalyst using a Training Set of Dually-Reviewed Citations from a Review-of-Review and Randomly-Selected Citations with Labels from Full Text Screening: Outcome Metrics for Machine Learning Algorithm Only. Appendix 4. Cohort Studies Missed by Review of Reviews Approach. Appendix 5: Table AbstrackR Outcomes for Each Training Set.

Acknowledgements Not applicable 


\section{Authors' contributions}

All authors participated in the traditional systematic review and review of reviews. SP performed semi-automation tests. SR, SP, and MV analyzed and interpreted the results of the semi-automation tests. SR was a major contributor in writing the manuscript. All authors read, revised, and approved the final manuscript.

\section{Funding}

Patient-Centered Outcomes Research Institute. (Task Order \#11 RTI.EVIDENCEMAPAMPTESP)

\section{Availability of data and materials}

The datasets during and/or analyzed during the current study available from the corresponding author on reasonable request.

\section{Ethics approval and consent to participate}

Not applicable

\section{Consent for publication}

Not applicable

\section{Competing interests}

The authors declare that they have no competing interests.

\section{Author details}

'RTI International, 307 Waverly Oaks Road, Suite 101, Waltham, MA 02452 USA. ${ }^{2}$ RTI International, 3040 East Cornwallis Road, Research Triangle Park, NC 27709, USA. ${ }^{3}$ UC Davis, Center for Healthcare Policy and Research, 2103 Stockton Blvd., Sacramento, CA 95817, USA.

Received: 13 February 2020 Accepted: 7 August 2020

Published online: 19 October 2020

\section{References}

1. Program EHC. Methods guide for effectiveness and comparative effectiveness reviews. Agency for Healthcare Research and Quality: Rockville, MD; 2014.

2. Methods Group of the Campbell Collaboration. Methodological expectations of Campbell Collaboration intervention reviews: conduct standards. Campbell Policies and Guidelines Series No. 3. https://www. campbellcollaboration.org/library/campbell-methods-conduct-standards. html. Published 2017. Accessed October 1, 2018

3. Institute of Medicine of the National Academies. Finding what works in health care: standards for systematic reviews. Washington, DC: Institute of Medicine of the National Academies; 2011.

4. National Cancer Institute. Cancer stat facts: prostate cancer. https://seer. cancer.gov/statfacts/html/prost.html. Published 2018. Accessed February 19, 2018.

5. Negoita S, Feuer EJ, Mariotto A, et al. Annual Report to the Nation on the Status of Cancer, part II: Recent changes in prostate cancer trends and disease characteristics. Cancer. 2018;124(13):2801-14. 29786851. https://doi. org/10.1002/cncr.31549.

6. Bell KJ, Del Mar C, Wright G, Dickinson J, Glasziou P. Prevalence of incidental prostate cancer: a systematic review of autopsy studies. Int J Cancer. 2015; 137(7):1749-57. 25821151. https://doi.org/10.1002/ijc.29538.

7. Fenton JJ, Weyrich MS, Durbin S, Liu Y, Bang H, Melnikow J. Prostate-specific antigen-based screening for prostate cancer: a systematic evidence review for the U.S. Preventive Services Task Force. Agency for Healthcare Research and Quality: Rockville, MD; 2018.

8. Sun F, Oyesanmi O, Fontanarosa J, Reston J, Guzzon T, Schoelles K. Therapies for clinically localized prostate cancer. Update of a 2008 systematic review. Agency for Healthcare Research and Quality: Rockville, MD; 2014

9. Ananiadou S. McNaught J. Text mining for biology and biomedicine Boston/London: Artech House; 2006.

10. O'Mara-Eves A, Thomas J, McNaught J, Miwa M, Ananiadou S. Using tex mining for study identification in systematic reviews: a systematic review of current approaches. Syst Rev. 2015:4:5. 25588314. https://doi.org/10.1186/ 2046-4053-4-5.
11. Wallace BC, Trikalinos TA, Lau J, Brodley C, Schmid CH. Semi-automated screening of biomedical citations for systematic reviews. BMC Bioinformatics. 2010;11:55. 20102628. https://doi.org/10.1186/1471-2105-11-55.

12. National Centre for Text Mining. RobotAnalyst. http://www.nactem.ac.uk/ robotanalyst/. Published 2016.

13. Przybyla P, Brockmeier AJ, Kontonatsios G, et al. Prioritising references for systematic reviews with RobotAnalyst: a user study. Res Synth Methods. 2018;9(3):470-88. 29956486. https://doi.org/10.1002/jrsm.1311.

14. Wallace BC, Small K, Brodley CE, Lau J, Trikalinos TA. Deploying an interactive machine learning system in an evidence-based practice center: abstrackr. Proceedings of the 2nd ACM SIGHIT International Health Informatics Symposium (IHI '12). New York, NY: ACM; 2012:819-824.

15. Gates A, Johnson C, Hartling L. Technology-assisted title and abstract screening for systematic reviews: a retrospective evaluation of the Abstrackr machine learning tool. Syst Rev. 2018;7(1):45. 29530097. https://doi.org/10. 1186/s13643-018-0707-8

16. Rathbone J, Hoffmann T, Glasziou P. Faster title and abstract screening? Evaluating Abstrackr, a semi-automated online screening program for systematic reviewers. Syst Rev. 2015;4:80. PMID: 26073974. doi: 10.1186/ s13643-015-0067-6.

17. Effective Health Care. Rapid review guidance document. https://www.ahrq. gov/sites/default/files/wysiwyg/funding/contracts/epc-vi/22-rapid_ evidence_products_guidance.pdf. Published.

18. GRADE Working Group. Welcome to the GRADE working group. http:// www.gradeworkinggroup.org/. Published n.d.

19. O'Connor AM, Tsafnat G, Gilbert SB, Thayer KA, Wolfe MS. Moving toward the automation of the systematic review process: a summary of discussions at the second meeting of International Collaboration for the Automation of Systematic Reviews (ICASR). Syst Rev. 2018;7(1):3. 29316980. https://doi.org/ 10.1186/s13643-017-0667-4.

20. Marshall IJ, Wallace BC. Toward systematic review automation: a practical guide to using machine learning tools in research synthesis. Syst Rev. 2019; 8(1):163. 31296265. https://doi.org/10.1186/s13643-019-1074-9.

21. Shekelle PG, Shetty K, Newberry S, Maglione M, Motala A. Machine learning versus standard techniques for updating searches for systematic reviews: a diagnostic accuracy study. Ann Intern Med. 2017;167(3):213-5. 28605762. https://doi.org/10.7326/L17-0124.

22. Gartlehner G, Wagner G, Lux L, Affengruber L, Dobrescu A, KaminskiHartenthaler A, Viswanatha M. Assessing the accuracy of machine-assisted abstract screening with DistillerAl: a user study. Syst Rev. 2019;277(8). PMID: 31727159. https://doi.org/10.1186/s13643-019-1221-3.

\section{Publisher's Note}

Springer Nature remains neutral with regard to jurisdictional claims in published maps and institutional affiliations.

Ready to submit your research? Choose BMC and benefit from:

- fast, convenient online submission

- thorough peer review by experienced researchers in your field

- rapid publication on acceptance

- support for research data, including large and complex data types

- gold Open Access which fosters wider collaboration and increased citations

- maximum visibility for your research: over $100 \mathrm{M}$ website views per year

At BMC, research is always in progress.

Learn more biomedcentral.com/submission 